\title{
High-Throughput Scaffold System for Studying the Effect of Local Geometry and Topology on the Development and Orientation of Sprouting Blood Vessels
}

\author{
Ariel A. Szklanny, Lior Debbi, Uri Merdler, Dylan Neale, Ayse Muñiz, Ben Kaplan, \\ Shaowei Guo, Joerg Lahann, and Shulamit Levenberg*
}

Live tissues require vascular networks for cell nourishing. Mimicking the complex structure of native vascular networks in vitro requires understanding the governing factors of early tubulogenesis. Current vascularization protocols allow for spontaneous formation of vascular networks; however, there is still a need to provide control over the defined network structure. Moreover, there is little understanding on sprouting decision and migration, especially within 3D environments. Here, tessellated polymer scaffolds with various compartment geometries and a novel two-step seeding protocol are used to study vessel sprouting decisions. Endothelial cells first organize into hollow vessels tracing the shape contour with high fidelity. Subsequent sprouts emerge in specific directions, responding to compartment geometry. Time-lapse imaging is used to track vessel migration, evidencing that sprouts frequently emerge from the side centers, mainly migrating toward opposing corners, where the density of support cells (SCs) is the highest, providing the highest levels of angiogenic factors. SCs distribution is quantified by smooth muscle actin expression, confirming the cells preference for curved compartment surfaces and corners. Displacements within the hydrogel correlate with SCs distribution during the initial tubulogenesis phase. This work provides new insight regarding vessel sprouting decisions that should be considered when designing scaffolds for vascularized engineered tissues. nor can metabolic waste be eliminated. ${ }^{[2]}$ The microvasculature (i.e., arterioles, capillary, and venules) is a key part of the vascular system, responsible for transferring nutrients from the blood stream into the tissues. For the past decade, tissue vascularization has been widely studied; the most prevalent approach involves coculturing endothelial cells (ECs) and support cells (SCs, which can differentiate to mural cells) within a 3D matrix suitable for cell adhesion, proliferation, and self-assembly into randomly organized vessel networks. ${ }^{[2-6]}$ In coculture, the ECs reorganize to form tubular structures while the SCs provide mechanical support and deposit extracellular matrix (ECM) proteins. In addition, there is a cross-talk between the two cell types and release of signaling molecules that promote tubulogenesis, sprouting, and vessel migration and stabilization as well as differentiation and maturation of the SCs. ${ }^{[7]}$

The most common 3D systems in tissue engineering are hydrogels, porous scaffolds, or a combination of both. Combining porous scaffolds and hydro-

\section{Introduction}

Currently, fabricating vascularized tissues is one of the main challenges in the field of tissue engineering. ${ }^{[1]}$ Without a proper vascular network, the innermost parts of a tissue cannot receive the nutrients required for cellular growth and tissue assembly,

\footnotetext{
A. A. Szklanny, L. Debbi, U. Merdler, B. Kaplan, S. Guo, Prof. S. Levenberg Department of Biomedical Engineering

Technion - Israel Institute of Technology

Haifa 32000, Israel

E-mail: Shulamit@bm.technion.ac.il

D. Neale, A. Muñiz, Prof. J. Lahann

Department of Chemical Engineering and Biointerfaces Institute

University of Michigan

Ann Arbor, MI 48109, USA
}

The ORCID identification number(s) for the author(s) of this article can be found under https://doi.org/10.1002/adfm.201901335.

DOI: 10.1002/adfm.201901335 gels results advantageous because it offers a cell-friendly environment for cells to grow, and provides mechanical support for the whole engineered construct. ${ }^{[8,9]}$ Previous research studied the development of vessel networks on the surface of porous scaffolds with varying topographical features, or the de novo vessel formation behavior when ECs and SCs are seeded within hydrogels with different geometric designs. ${ }^{[10-13]}$ However, these works did not elucidate the dynamics affecting vascular network decisions regarding sprouting origin and vessel orientation within 3D structures. Understanding these results is imperative, especially with the rapid increase of scaffold fabrication techniques that permit fabrication of scaffolds with micrometer-scale precision, such as 3D jet writing, extrusionbased 3D printing, and stereolithography. ${ }^{[14-16]}$ This knowledge could help researchers design 3D environments that better suit their requirements for recreating a specific target tissue.

In this work, we investigated the factors governing the origin location of new sprouts, and the subsequent vessel migration and orientation in 3D systems. For this, we created 3D scaffolds 
with tessellated compartment geometries which, combined with a novel seeding technique, allowed us to control the initial spatial configuration of the newly formed vessels through tubulogenesis. We designed the scaffold compartments with varying geometries to shed light on the factors affecting the spatial origin of the sprouting vessels and their migration pattern. Further, we investigated the correlation between compartment geometry and SCs distribution, and its consequent effect on vessel migration. Finally, by tracking micrometric displacements within the hydrogel, we demonstrated that the SCs generate higher deformations in the vicinity of the compartment walls.

\section{Results}

\subsection{Scaffold Fabrication and Two-Step Cell Seeding}

Studying the complex 3D development of vascular networks requires a proper platform that enables direct visualization of the biological process. To create such a system, we developed a novel 3D two-step cell seeding protocol using tessellated scaffolds with highly defined and repeated compartments (Figure 1). The scaffolds were fabricated by coating a silicon wafer with OmniCoat, then spin-coating the wafer with SU-8 photoresist. The photoresist was cross-linked by illuminating its surface with UV light through custom-made negative masks (Figure 1Ai,ii). The masks were designed to produce 80 scaffolds per batch $(5.7 \mathrm{~mm} \times 5.7 \mathrm{~mm})$. The scaffolds had 121 compartments in the shape of circles, hexagons, squares, or 11 compartments in the shape of parallel rectangles (Figure 1Aiii, Figure S1, Supporting Information). To promote tubulogenesis, we used a novel 3D two-step cell seeding protocol. First, the scaffolds were coated with a fibronectin solution (50 $\mu \mathrm{g} \mathrm{mL} \mathrm{m}^{-1}$; Figure 1Aiv), an extracellular matrix (ECM) glycoprotein frequently used to promote cell attachment to different substrates. ${ }^{[17]}$ The first step of the cell seeding consisted of suspending $10^{5}$ ECs (here we used human adipose microvascular endothelial cells, HAMECs) in $25 \mu \mathrm{L}$ of cell medium and seeding them onto the fibronectin-coated scaffolds (Figure 1Av). The ECs attached and proliferated on the SU-8, until reaching confluence after 1-2 d (Figure 1Avi,vii). The second step of the cell seeding included suspending $10^{5}$ SCs (here we used dental pulp stem cells, DPSCs) in fibrin pre-gel solution $(25 \mu \mathrm{L})$ and adding it on top of the endothelialized scaffolds, filling the compartments (Figure 1Aviii). After fibrin gelation, the SCs continued proliferating and expanding through the gel, while the ECs detached from the SU-8 walls into the hydrogel and formed hollow tubes (Figure 1Aix). After 2 to $3 \mathrm{~d}$, new vessels sprouted from the circumvallating vessels into the gel (Figure 1Ax). Figure 1B shows scanning electron microscopy (SEM) images of the different stages of the protocol. This seeding protocol was developed to obtain vascular networks with a greatly controlled starting morphology, in response to scaffold geometry, recreating the compartment shape with high fidelity. Moreover, this system allowed us to produce highly repeatable vascular networks.

We demonstrated the importance of the cell seeding order on the organized vessel formation by reversing the ECs and
SCs seeding order, or simultaneously seeding both cell types suspended fibrin on fibronectin-coated scaffolds (Figure S2, Supporting Information). While the proposed seeding protocol $(\mathrm{ECs} \rightarrow \mathrm{SCs})$ resulted in an organized vessel network development with clearly recognizable tubulogenesis and sprouting phases (Figures S2 and S3, Supporting Information), the other groups ( $\mathrm{SCs} \rightarrow \mathrm{ECs}$ and $\mathrm{SCs}+\mathrm{ECs}$ ) resulted in a less organized development, starting with ECs cluster formation and subsequent thinning and rearranging into vessels, a known phenomenon shown in previous works. ${ }^{[18]}$

\subsection{Vascular Development Depends on Compartment Geometry}

Currently, porous scaffolds used for tissue engineering possess varying pore shapes and dimensions. We hypothesized that these parameters might affect the behavior of engineered vascular networks. We sought to understand the effect of these factors on the early stages of the vascular network development. For this, we designed scaffolds with different compartment geometries. The selected shapes were circles, hexagons, squares, and rectangles, sharing a common length $d$ (Figure 1Aiii). The rectangles were imaged and studied in the center of the compartment, far from the short sides, virtually resulting in a compartment only formed by two opposing walls. The characteristic length $d$ was selected based on the behavior of sprouting vessels in rectangular-compartmented scaffolds with increasing wall-to-wall distances (Figure S4, Supporting Information). We investigated wall-to-wall distances of 110 , 250, 500, and $1000 \mu \mathrm{m}$. Cells seeded on the $110 \mu \mathrm{m}$ scaffolds failed to form differentiable sprouts (data not shown), mostly forming structures that resembled endothelial cords, likely due to the limited available space. ${ }^{[10]}$ For the remaining geometries, we quantified the emerging and crossing sprouts at days 3, 5, and 7; scaffolds with $500 \mu \mathrm{m}$ gaps had the most emerging sprouts for all the time points. The number of vessels crossing the gap were higher for these scaffolds at day 3 and 7, while it was higher for the $250 \mu \mathrm{m}$ scaffolds at day 5 . To directly compare different compartment geometries, we chose $d=500 \mu \mathrm{m}$, since this gap size effectively promoted sprouting, and had a high amount $(>50 \%)$ of crossing vessels, suggesting interactions between support and endothelial cells located around neighboring walls (Figure S4, Supporting Information).

We applied the two-step seeding protocol on the four scaffold types, and imaged them at days $0,1,3,5$, and 7 (Figure 2A). Prior to support cell and hydrogel addition, the endothelial cells completely covered the scaffold walls (day 0). At day 1 , the endothelial cells started detaching from the walls, creating a layer around the compartment. Both tubulogenesis and sprouting phenomena were already observable by day 3; the endothelial cells organized into thick hollow vessels bordering the compartment walls, with thin sprouts and tip cells emerging into the gel. By day 5 and day 7, the outer vessels reduced their thickness, and the number of interconnected vessels greatly increased. Then, we quantified the vascular network development for the different cases using Angiotool, a software that analyzes vascular network images and returns several characteristic parameters (Figure S5, Supporting Information). ${ }^{[19]}$ To compare between the different shapes, we focused only 
A

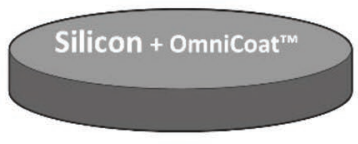

i. Wafer preparation

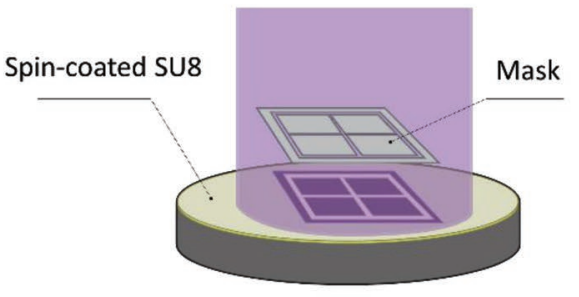

ii. SU8 UV cross-linking $d=500 \mu \mathrm{m}$

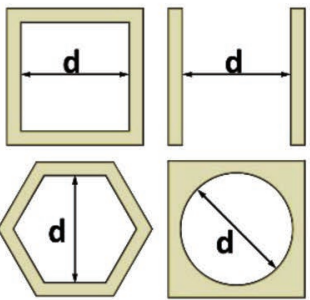

iii. Designed geometries

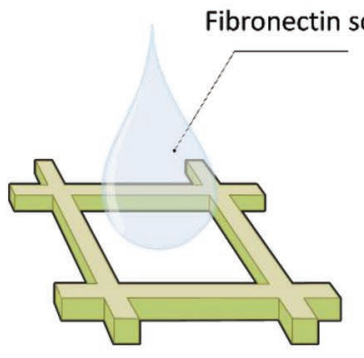

iv. Fibronectin coating

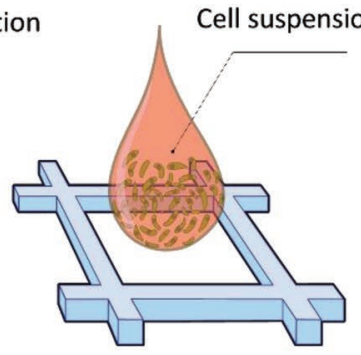

v. Endothelial cell seeding

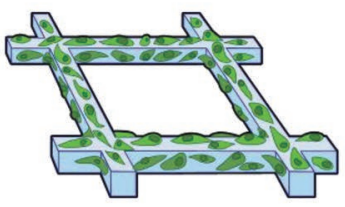

vi. Endothelial cell attachment

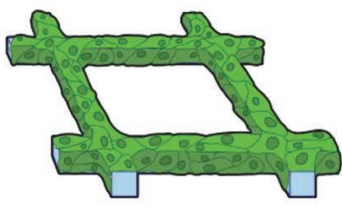

vii. Endothelial cell on-scaffold growth

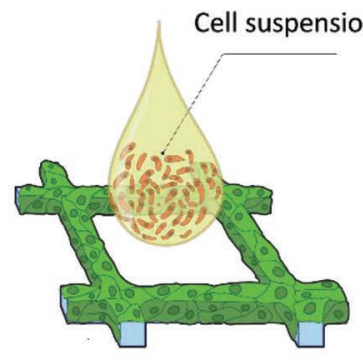

viii. Support cell seeding

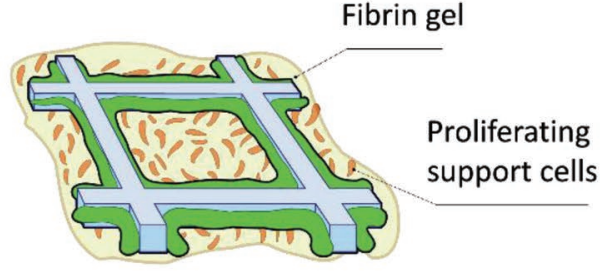

ix. Tubulogenesis

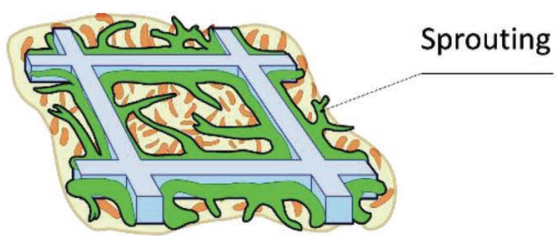

x. Sprouting

B
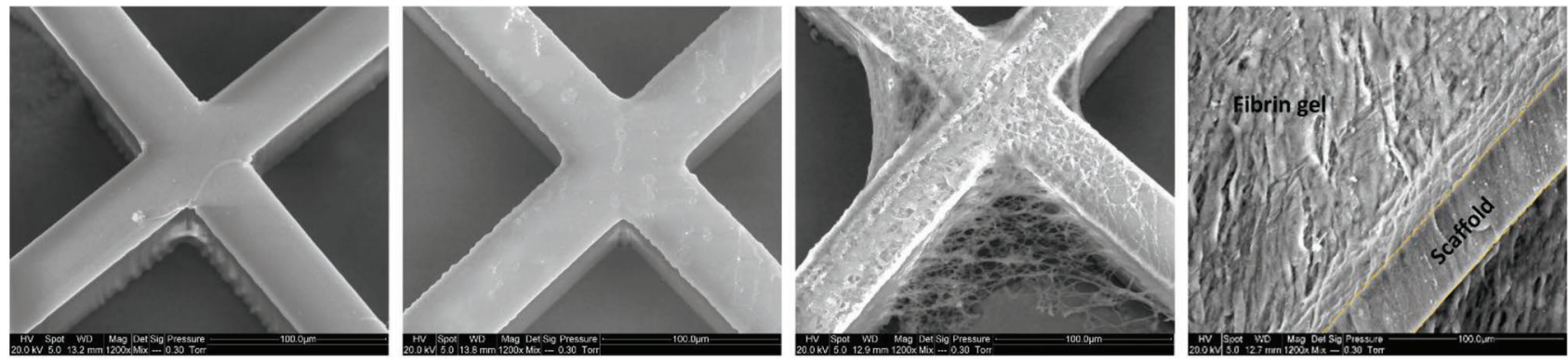

Figure 1. Schematic of scaffold fabrication and cell seeding. A) Scaffolds are prepared (i) by coating a silicon wafer with OmniCoat, then (ii) spin-coating SU8 on it, crosslinking it with UV light through a negative mask, and washing the remaining SU8. (iii) The resulting scaffolds have different compartment geometries: squared, hexagonal, circular, or rectangular. (iv) Scaffolds are coated with a fibronectin solution, (v) then seeded with endothelial cells suspended in medium, (vi) which attach to the scaffold walls. (vii) The endothelial cells are cultured until they fill the scaffold. (viii) The supporting cells are suspended in fibrin gel, and seeded on top of the endothelial cells filled scaffolds. (ix) The constructs are cultured, and the endothelial cells start the tubulogenesis phenomenon next to the walls of the scaffold. (x) This results in new sprouts emerging from the vessels into the compartments. B) SEM images of (i) SU8 scaffold, (ii) fibronectin-coated scaffold, (iii) endothelial-seeded scaffold, and (iv) fibrin-seeded scaffold. The dashed yellow line represents the SU-8 scaffold wall.

on the vessels located within the compartments and analyzed their total vessel length and area (Figure 2B, left and center; Table S2, Supporting Information). For the rectangular scaffolds, both vessel area and length increased linearly, the latter having a slightly steeper slope. In contrast to the rectangles, the circle also commenced growing at the first stages, but 
A Day 0
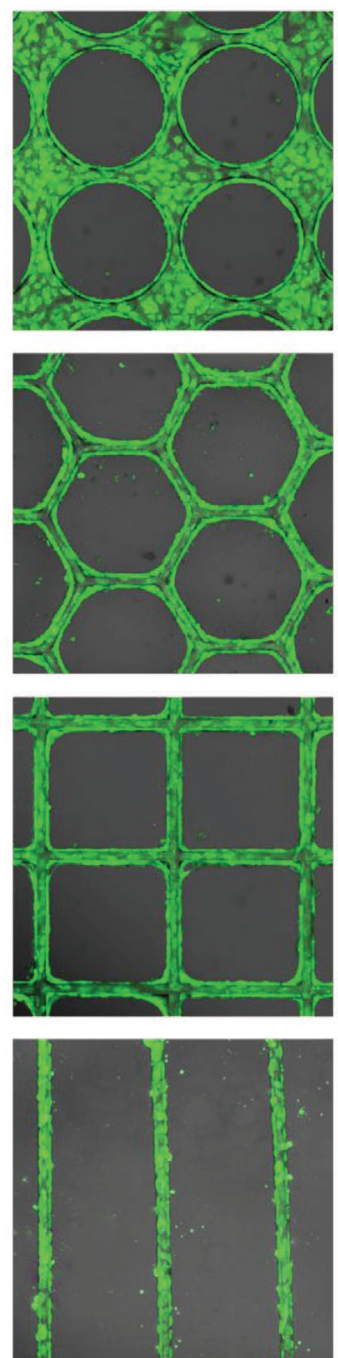

Day 1
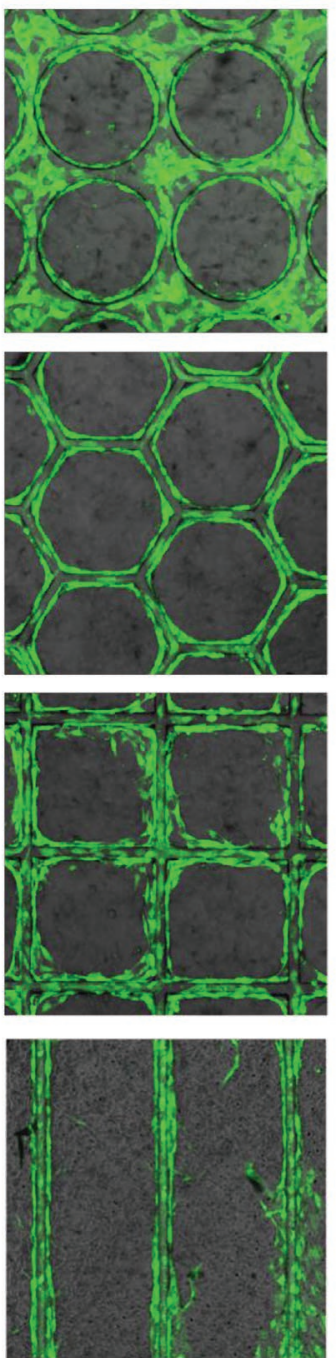

Day 3
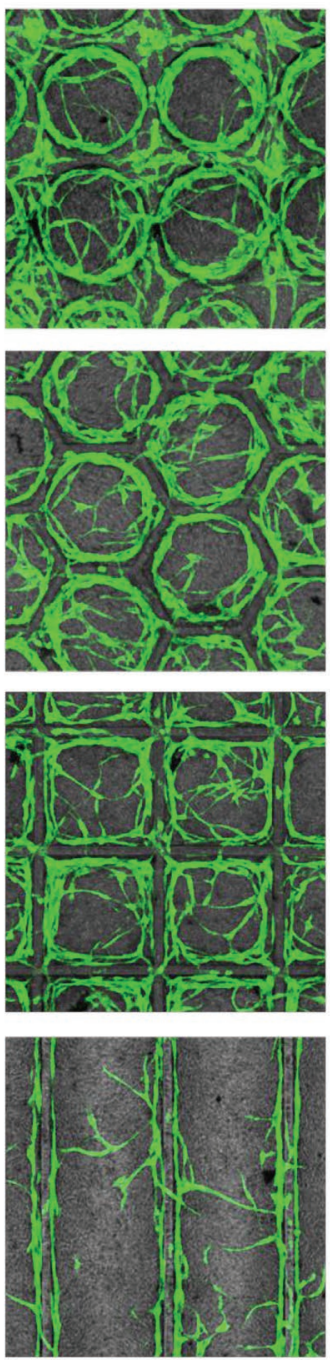

Day 5
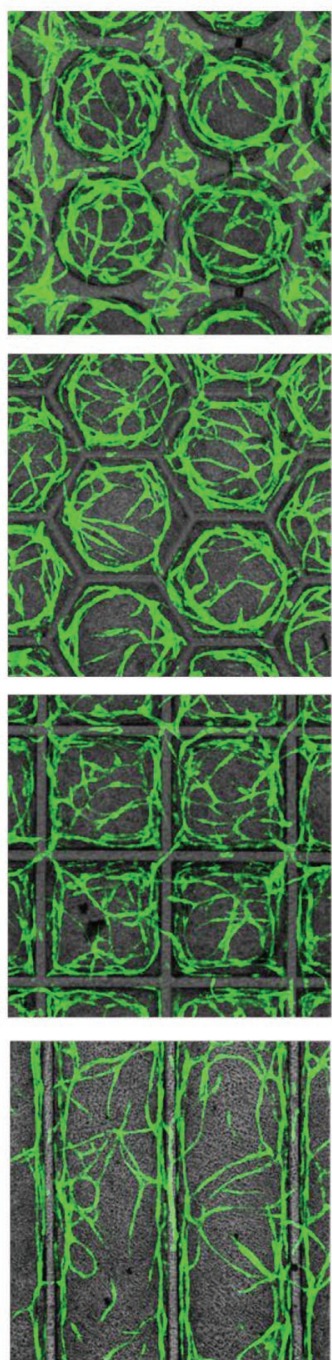

Day 7
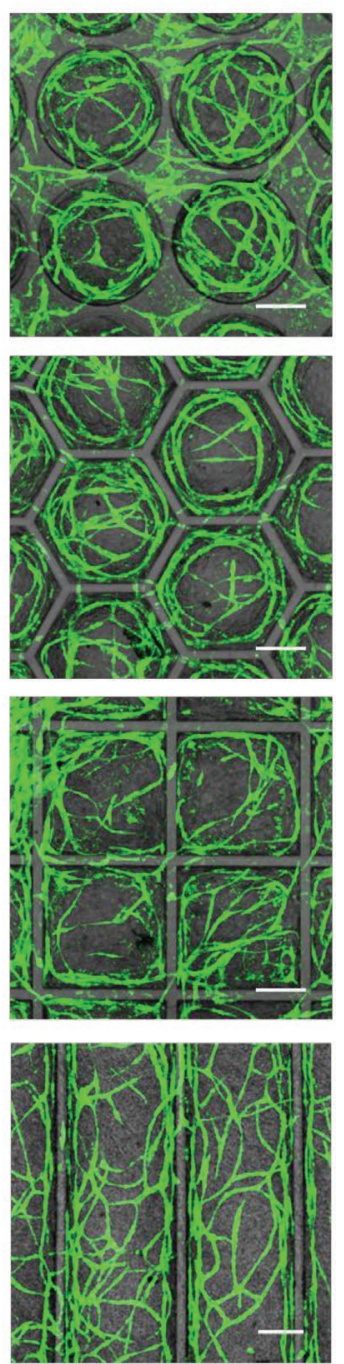

B

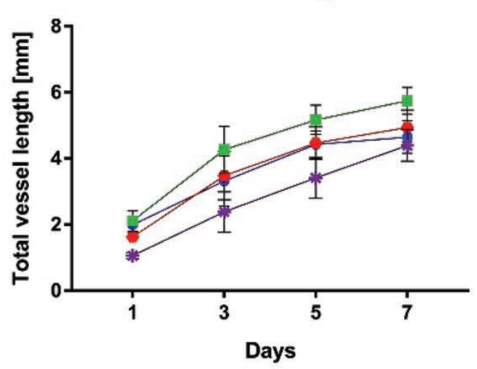

Vessel Area

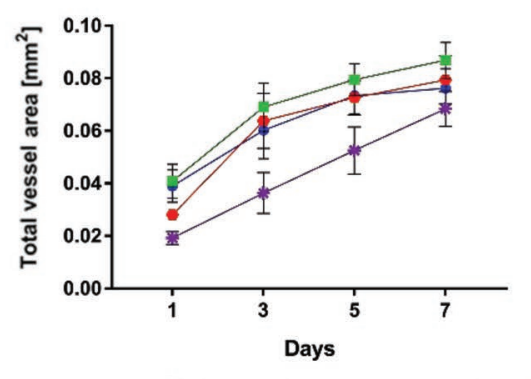

- Circle - Hexagon $\rightarrow$ - Square
Normalized Vessel Thickness

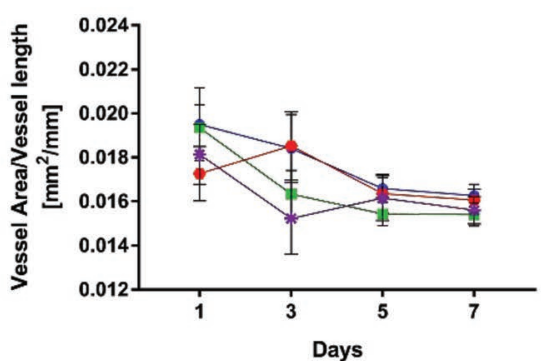

Figure 2. Vascular network development dynamics in ECs-SCs seeded scaffolds. A) Representative confocal images of the vascular development within different compartment geometries for several time points: days 0, 1, 3, 5, and 7. Day 0 images represent the ECs (green) attached to the fibers before the SCs addition. Day 1 and after consist of scaffolds containing both cell types (scale bar: $200 \mu \mathrm{m}$ ). B) Analysis of key parameters of vascular development: left: total vessel length; center: total vessel area; right: normalized vessel thickness ( $n>5$; ns: nonsignificant).

drastically decreased the growth rate between days 5 and 7 , almost reaching a plateau. This behavior is likely related to lack of available space, as the networks are greatly condensed at day 7 (Figure 2A). Both, hexagon- and square-shaped scaffolds, supported increased vascular development during the earlier stages, but showed reduced growth rates at the latter days. 
As with the circles, we attribute the decreased growth rates to space constrains. Nonetheless, by day 7, circles, hexagons, and squares reach similar values of vessel length and area. This can be explained by the area-to-perimeter ratio $(A / P)$. The $A / P$ describes the ratio between the available hydrogel volume for cell growth and development (represented by the area, A) and the initial ECs number attached to the compartment walls (represented by the perimeter, $P$ ). We expected a similar development within the hexagonal, circular, and squared shapes since they share the same $A / P\left(125 \mu \mathrm{m}^{2} \mu \mathrm{m}^{-1}\right)$. In contrast, vessels within the rectangular scaffolds did not reach a plateau within one week. We anticipated these different trends since the $A / P$ in the rectangular shapes is almost twice as high as the $A / P$ of the other shapes $\left(225 \mu \mathrm{m}^{2} \mu \mathrm{m}^{-1}\right)$. Logically, the higher available space in the rectangular shapes enabled the vessels to continue developing without reaching a plateau after a week, in contrast to the remaining shapes. Additionally, we analyzed the average vessel thickness by calculating the total vessel area/length ratio. At day 1 , when endothelial cells only start detaching and organizing next to the walls, the vessel average thickness had similar values for all shapes. On day 3 , the rectangles showed the thinnest vessels $(15.23 \pm 1.62 \mu \mathrm{m})$, followed by the squares $(16.33 \pm 1.08 \mu \mathrm{m})$, hexagons $(17.89 \pm 0.69 \mu \mathrm{m})$, and circles $(18.41 \pm 1.54 \mu \mathrm{m})$. Interestingly, the increasing vessel thickness has the same order as the shapes' isoperimetric quotient (or circularity; Table S1, Supporting Information), defined as $C=4 \pi \cdot A \cdot P^{-2}$, where $A$ is the shape area, and $P$ is the shape perimeter. Much like the vessel thickness, the $C$ values followed an increasing order for the squares (0.7854), hexagons (0.9069), and circles (1). The rectangular compartments are nonclosed curves and lack a $C$ value. Vessel thickness for the later stages of development was remarkably similar on all the geometries, resulting in nonsignificant differences for all the shapes at day 7 (Table S2, Supporting Information). These results suggest that compartment geometry does influence the vessel morphology at early stages of vascular development. Nonetheless, it is important to emphasize that although the spaces between the compartments are noticeably different among the geometries (i.e., having varying surfaces with differing attached cells numbers), we demonstrated that the cells located on the flat walls adjacent to the compartments do not influence the development of the vessels located within the compartments (Figure S6, Supporting Information).

\subsection{Vessel Orientation Is Affected by Compartment Geometry}

To further characterize the influence of compartment shape on vessel networks, we quantified the vessel orientation within the compartments. For this, we obtained confocal images of the vessel networks and performed a computer analysis consisting of (i) skeletonizing the image, (ii) codifying each separate element (vessel branch) according to the angle of their major axis length, (iii) accumulating the length of all the elements in bins of $5^{\circ}$ (e.g., adding the total length of all the elements having an angle between $55^{\circ}$ and $60^{\circ}$ ), and (iv) plotting the obtained distribution in a range from $0^{\circ}$ to $90^{\circ}$ (Figure S7, Supporting Information). Due to the shapes symmetry along the $x$-axis, we unified the distribution according to the angles' absolute value (e.g., elements with $-82^{\circ}$ and $-84^{\circ}$ would be binned together).

To quantify the orientation of the new sprouts within the compartment, we first considered the effect of the vessels adjacent to the scaffold walls, since these vessels were present from day 1 , and appeared ubiquitously in every compartment, regardless of the shape. To eliminate this effect, we analyzed confocal images for day 1 (Figure 3A); the color-coded images show the elements angle in a $90^{\circ}$ range, as explained above. As expected, the plots accurately describe the characteristic angles of the scaffolds, showing a fairly homogeneous distribution along the range for the circle, two peaks at $0^{\circ}$ and $60^{\circ}$ for the hexagon, two peaks at $0^{\circ}$ and $90^{\circ}$ for the squares, and a single peak at $90^{\circ}$ for the rectangles. While the data for day 1 do not strictly represent the orientation of hollow vessels, these peaks were fairly conserved after the tubulogenesis and sprouting phenomena occurred (Figure S8, Supporting Information), resulting in data used for the normalization of the later time points. After accounting for the effects of the circumvallating vessels, we were able to compute the true effect of the compartment geometries on the sprouting vessels orientation; the polar plots represent the fold change of the total vessel lengths compared to day 1 (Figure 3B, top). For each shape, the angle distribution remained mostly unchanged for all time points. The circular compartments presented a highly homogeneous distribution in all directions, with the difference between the maximum and minimum peaks (peak-to-peak difference) of $65 \%$ at day 7 , in relation to the minimum value (i.e., peak-to-peak $=$ $\left.100 \% \times \frac{\text { peak }_{\text {maximum }}-\text { peak }_{\text {minimum }}}{}\right)$. The hexagonal shape showed peak $_{\text {minimum }}$

peaks around $15^{\circ}, 40^{\circ}, 45^{\circ}, 75^{\circ}$, and $80^{\circ}$, with a day 7 peak-topeak difference of $348 \%$. The squared compartments showed peaks around $15^{\circ}, 30^{\circ}, 60^{\circ}$, and $75^{\circ}$, having a peak-to-peak variation of $210 \%$ at day 7 . For the rectangular compartmented scaffolds, the main peak was located around $60^{\circ}$, with a peakto-peak difference of $324 \%$. The lower peak-to-peak percentage of the circles, compared to those of the other shapes, suggests that circular symmetry does not encourage the vessels to follow a preferred direction. On the other hand, the other shapes did show specific vessel network orientation. To understand the occurrence of these prominent peaks, we thoroughly inspected the analyzed images, finding a link between the angles and the geometries. While the circles showed a uniform distribution, the peaks for the hexagons and squares were much correlated to line segments connecting the center of the sides with the opposing corners (Figure 3B, bottom). On the other hand, the peak of the rectangular compartments can be explained by an optimization model of vascular tree structures of Murray's law, developed by Uylings. ${ }^{[20]}$ The model describes the bifurcation angle for sprouting vessels, based on the parent and daughter relative vessels diameter. The vessels adjacent to the wall in the rectangular scaffolds presented a daughter/mother vessel diameter of $\approx 0.5$ which, like our results showed, represents an angle of $57.9^{\circ}$ within the model. The surrounding peaks might be explained by the intrinsic variability of the bifurcation mechanism. ${ }^{[21]}$

As showed above, for all the shapes, the total vessel length was the smallest at day 3 (Figure 2B); nonetheless, the main orientation peaks were already present (Figure 3B, top). To 
A

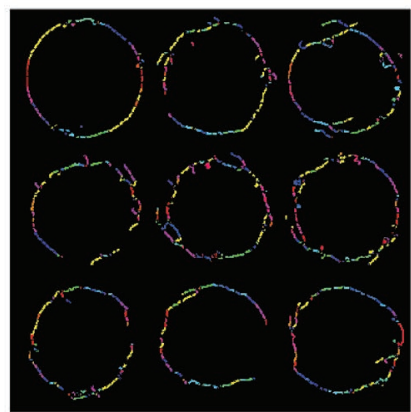

$90^{\circ}$

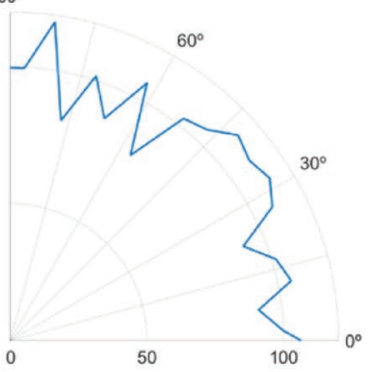

Hexagon

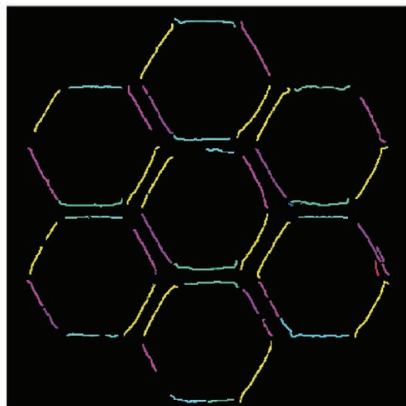

$90^{\circ}$

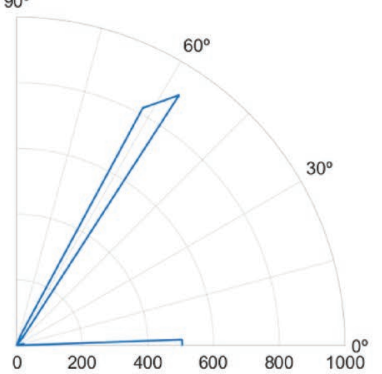

Square

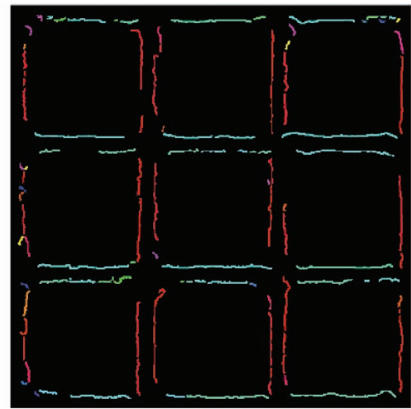

$90^{\circ}$

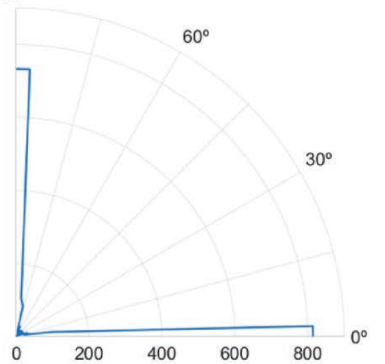

Rectangle

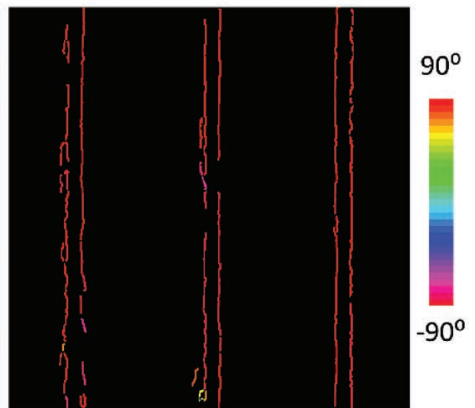

$90^{\circ}$

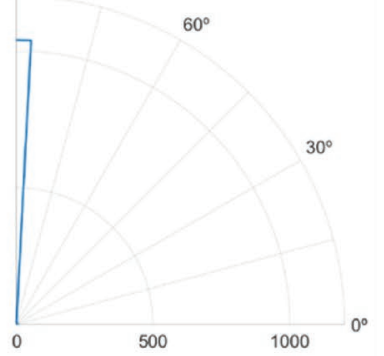

Day 1 - Total vessel length $[\mu \mathrm{m}]$

B $90^{\circ}$

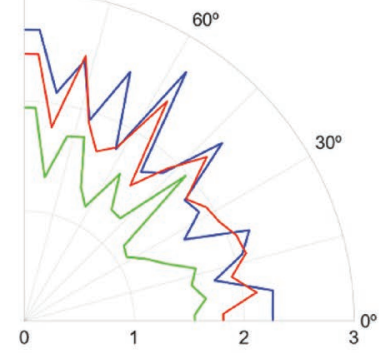

Hexagon

$90^{\circ}$

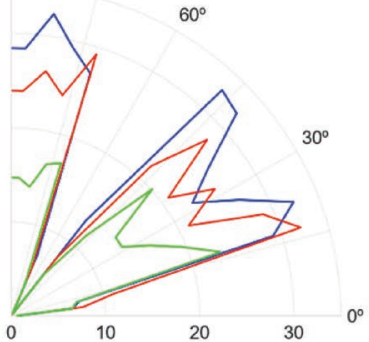

Square

$90^{\circ}$

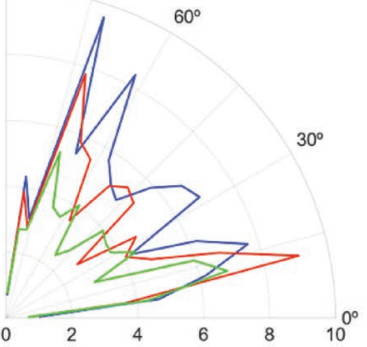

Normalized vessel length [fold change]
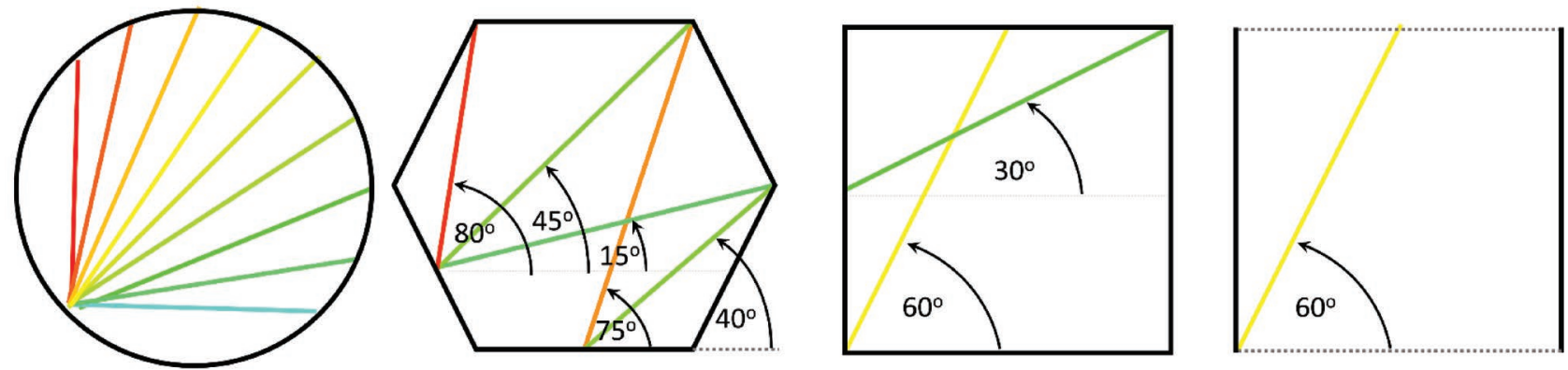

Figure 3. Vessel total length angular distribution. A) Vessel orientation analysis at day 1. Top: Color-coded skeletonized images. Each separate vessel branch (element) is colored according to the angle of its major length axis. Bottom: Angle distribution of total vessel length for the different geometries. Total length is represented as the radial distance from the origin to the outmost peaks for every angle $(n>3)$. B) Normalized angle distribution of total vessel length for the scaffolds at days 3,5 , and $7(n>3)$. The data were normalized to day 1 to reduce the presence of the vessels adjacent to the wall and better show the effect of scaffold topography on the orientation of new sprouts within the compartments. Bottom: The inferred peaks orientation related to the compartments geometry. The lines are colored according to the angular scale in (A). 
comprehend the vessel behavior behind the general network alignment, we performed time-lapse imaging to separately track sprouts emerging from the surrounding vessels after the tubulogenesis phenomenon (Figure 4; Movie S1, Supporting Information). We performed the two-step seeding as usual; however, as ECs, we seeded HAMEC-ZsGreen (green), HAMEC-TdTomato (red), and DiD-stained HAMEC (blue) in equal amounts $\left(3.3 \times 10^{4}\right.$ cells of each type), facilitating manual identification and tracking of single vessels. Time-lapse continuous imaging allowed us to identify the first sprouting vessels. First, we observed the delay differences for the first sprouts to form in relation to compartment shape. The time taken for the first sprout to appear was, once again, in accordance with the shape circularity. The average times were $55 \pm 0.79 \mathrm{~h}$ for circles, $50 \pm 2.17 \mathrm{~h}$ for rectangles, $45.6 \pm 1.98 \mathrm{~h}$ for hexagons, and $32.8 \pm 2.81 \mathrm{~h}$ for squares (Figure $4 \mathrm{~A}$ ). The rectangular compartment does not possess circularity, being a nonclosed curve; however, like the circular compartments (and unlike the polygonal shapes), it presents no discontinuities in its perimeter. Published mathematical models suggest that local ECM anisotropy promotes increased cell migration. ${ }^{[22]}$ We hypothesize that the discontinuity between the corners and sides of the polygonal shapes may generate ECM discontinuities that could explain the faster ECs-vessel sprouting from its walls into the compartment center, in contrast with the ECs in the circular and rectangular compartments that may perceive a more uniform matrix along the compartment wall. Additionally, we investigated the development behavior of the vessel network at the earliest stages, quantifying the total vessel length for the shapes from the time-lapse imaging (Figure 4B). Vessel lengthening showed two differentiated stages, separated by the first sprout mark in the graphs (dotted line). Before the first sprout, all the shapes showed an almost steady total vessel length value. This resulted from the endothelial cells detaching from the walls to form tube-like structures, in which the cells do not spread in length, but form thicker structures (Movie S1, Supporting Information). Once the tubulogenesis was more advanced, the newly formed vessels gave place to new sprouts, which emerged and migrated to different areas within the compartment. The length growth can be observed in the graphs as an increasing total vessel length, after the first sprout mark. The real-time imaging, quantification, and analysis proves the great potential of our system to monitor the development of vascular networks, and the capability of identifying different vessel behavior in response to specific factors or events.

Next, we sought to verify our hypothesis on vessels connection between the compartment corners and side's center (Figure 3B, bottom). To investigate the sprouting localization and vessel migration, we quantified and calculated the percentages of new sprouts starting from the side center, the percentage of vessels reaching the compartments, and the percentage of the vessels both departing from a side center and arriving at an opposing corner, referred here as on-track vessels (Figure 4C). The on-track vessels for the rectangular compartments were considered as those having a track angle between $30^{\circ}$ and $60^{\circ}$. The circles, lacking sides and corners, were not included in our calculations. Furthermore, the vessels within this compartment geometry migrated in seemingly arbitrary directions, in accordance with the angular distribution shown in Figure 3B. The percentage of vessels departing from a side center for the hexagon and square was 71 and $67 \%$, while the percentage of vessels arriving at a corner was 82 and $73 \%$, respectively. As for the on-track vessels, the percentages were 68, 60, and 64\%, for the hexagons, squares, and rectangles. Figure 4D shows examples of paths displayed by on-track vessels, and randomly distributed paths for the circular compartments. Furthermore, we quantified and compared the velocity of vessels migrating toward the corners within the close-shaped compartments (Figure S9, Supporting Information). By measuring the migration velocity of sprouts within the hexagonal compartments, we observed that when reaching corners located nearer or farther away, the movement velocity remained the same. However, when comparing the migration speed among the different geometries, we observed that the mean velocities ranked according to the shape circularity; vessels in the square compartments move at the slowest mean velocity $\left(12.39 \pm 2.61 \mu \mathrm{m} \mathrm{h}^{-1}\right)$, while those in the circular compartments moved with the highest migration speed $\left(18.98 \pm 1.78 \mu \mathrm{m} \mathrm{h}^{-1}\right)$. We suspected that the high percentages of the measured parameters and the difference in velocities were related to the support cells location, thus we proceeded to characterize their distribution within the varying compartment shapes.

\subsection{Location and Accumulation of Support Cells Govern Sprouting Orientation}

The transmitted light channel in the time-lapse imaging (Movie S2, Supporting Information) revealed a widening mass of cells proliferating next to the compartment walls and, in particular, in the corners of the hexagonal and squared compartments. We observed that the fluorescent endothelial cells did not form the totality of the thickening cell layer. Previous works have shown that fibroblast-like cells adhere and proliferate at higher rates on stiffer substrates. ${ }^{[23]}$ Our system is composed of two materials with highly distinct characteristics, the SU-8 walls and the fibrin gel, with Young's moduli in the order of the GPa and $\mathrm{kPa}$, respectively. ${ }^{[24]}$ Therefore, we concluded that part of the SCs accumulated on the stiff scaffolds wall after being seeded, and proliferated more rapidly, creating a noticeable cell buildup. To confirm and quantify this, we stained fixed scaffolds for $\alpha$-smooth muscle actin (SMA), a cytoskeleton protein expressed by these SCs (SCs-SMA+), especially when surrounding and stabilizing vessels. ${ }^{[4]}$ We analyzed the support cell accumulation by imaging the vessel networks within the compartments, focusing on the SMA expression (Figure 5A). Thresholding the signal by intensity, we created binary images of the SMA adjacent to the walls and proceeded to measure its thickness, from the innermost noticeable signal to the wall surface. The thickness was measured radially, from the center of the compartment outward, facilitating its characterization related to the compartment shape. Confocal images showed an evident difference among the shapes (Figure 5B, left), displaying a thicker layer for higher shape circularity factors (Table S1, Supporting Information), both at days 3 and 7. Day 7 images revealed a denser layer, with SCs further accumulating, presenting a rounder innermost shape, a behavior shown in previous works. ${ }^{[25]}$ Tessellated scaffolds with rectangular 
A

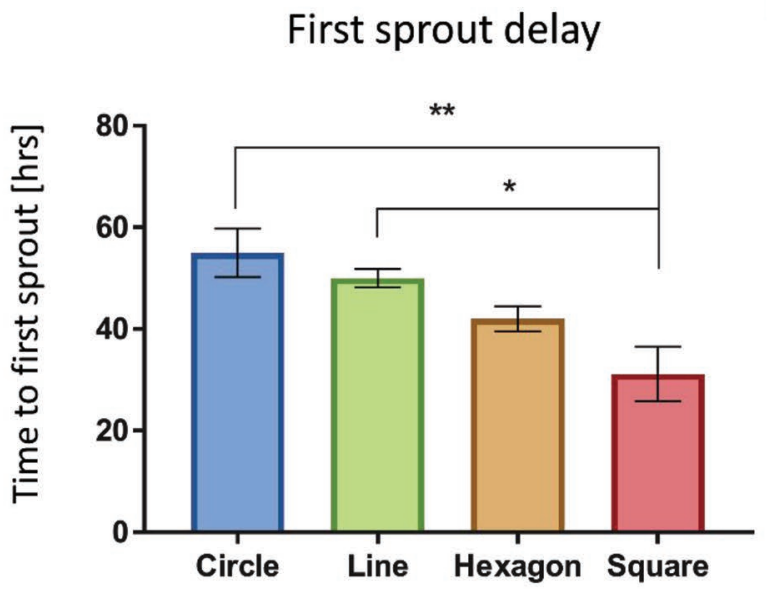

B

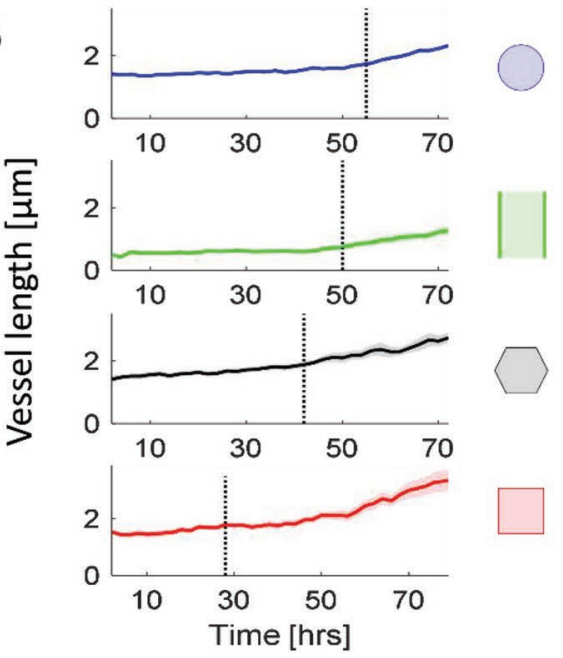

C

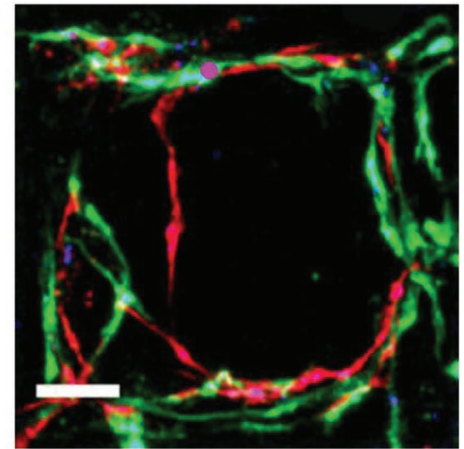

$\mathrm{t}=42 \mathrm{~h}$

D

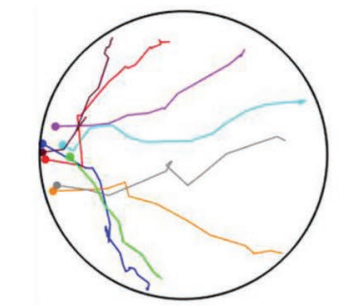

Departing from side center

Reaching a corner

On-track vessels

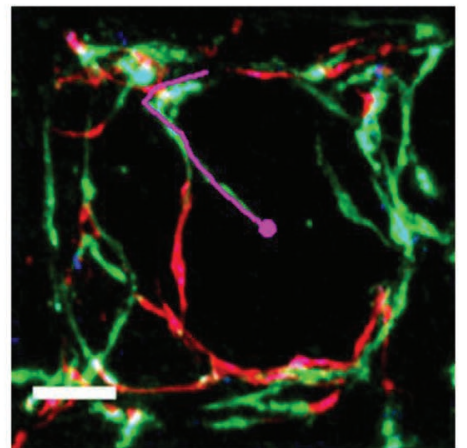

$\mathrm{t}=50 \mathrm{~h}$

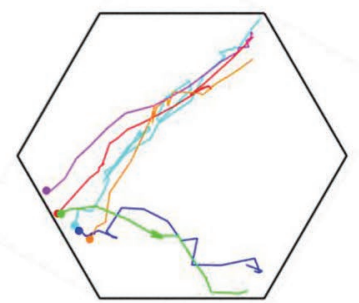

$71.42 \%$

$82.14 \%$

$67.85 \%$

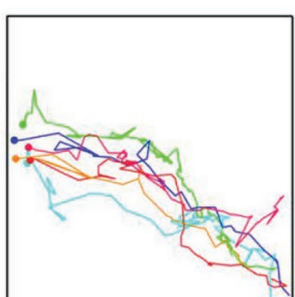

$66.67 \%$

$73.33 \%$

$60.12 \%$

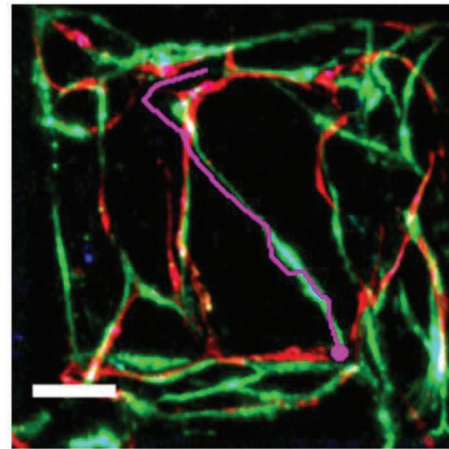

$\mathrm{t}=60 \mathrm{~h}$

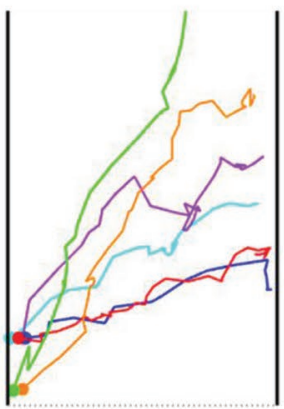

$64.27 \%$

Figure 4. Real-time imaging of sprouting vessels in geometrical compartments. A) Delay of first sprout appearance within the geometrical compartments $(* p<0.05, * p<0.01 ; n>5)$. B) Measurement of vessel lengthening in real time. The development is separated into two stages demarked by the first sprout delay (dotted line): before the first sprout, a vessel assembly and organization phase, and after, a sprouting phase, resulting in a continuous overall network lengthening. C) Representative images of new sprout tracking. The magenta dot shows the advance front of the vessel (red, HAMEC-TdTomato; green, HAMEC-ZsGreen; blue, DiD-stained HAMEC), and the magenta line indicates the path it followed. D) Examples of paths followed by on-track vessels, for each of the shapes. Each color represents a different vessel, and the dots indicate the starting point of each path. The tabulated values show for every geometry (except for the circles) the average percentage of all new sprouts departing from the center of a side, of new sprouts reaching a corner, and of the on-track vessels, which departed from the center, and reached a corner (for hexagon and square) or sprouted with an angle between $30^{\circ}$ and $60^{\circ}$ for the rectangles $(n>12$ ).

compartments showed only a thin layer of SMA-expressing cells, with apparent small to inexistent differences between the time points. Further quantification confirmed our observations
(Figure 5B). Scaffolds with rectangular compartments presented a uniform thickness along the walls with a mean value of $7.41 \pm 1.04 \mu \mathrm{m}$ for day 3 , and $13.61 \pm 0.99 \mu \mathrm{m}$ for day 7 . Both 
A

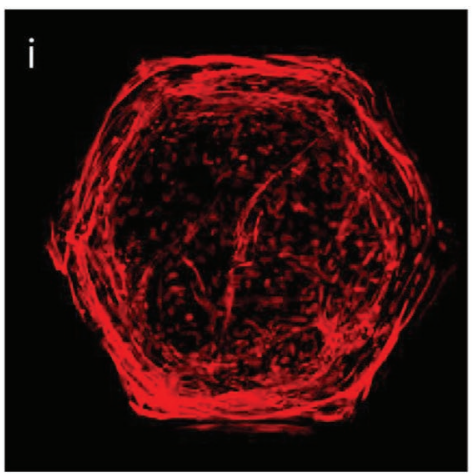

B
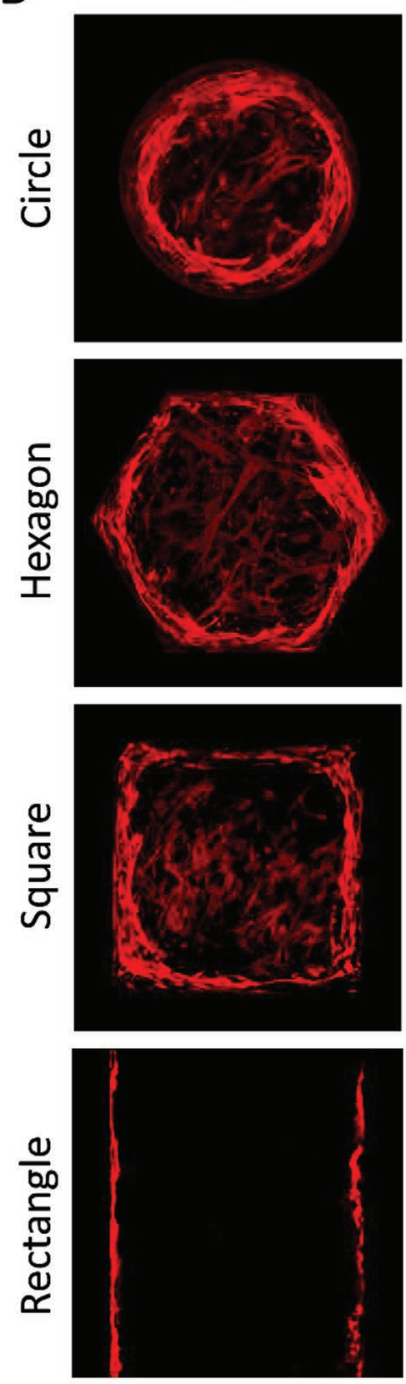

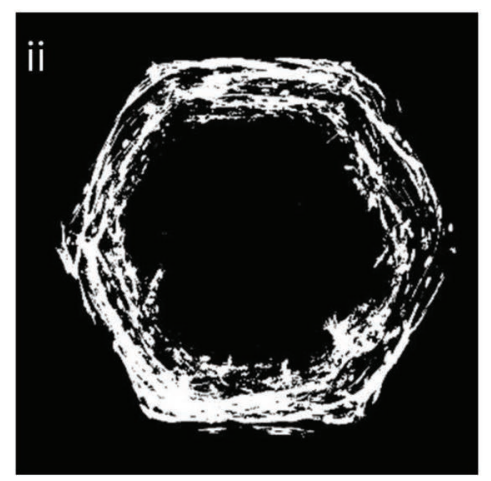

Day 7
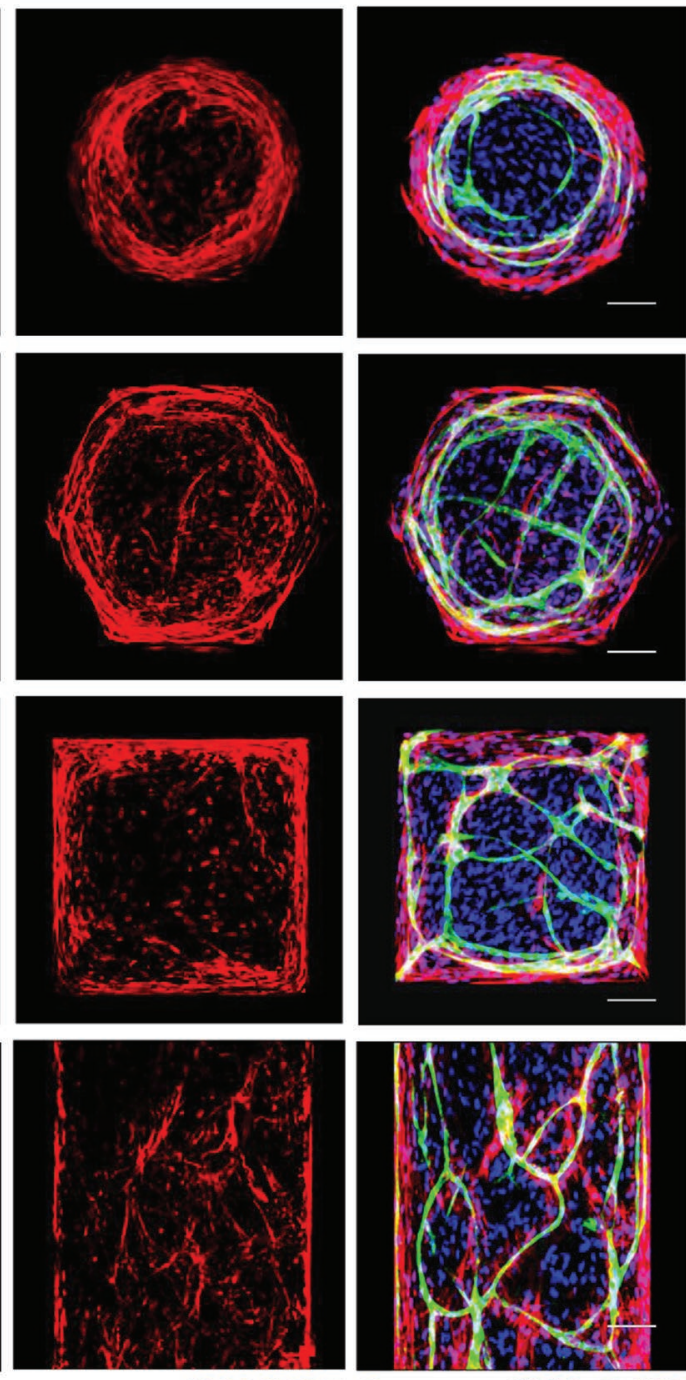

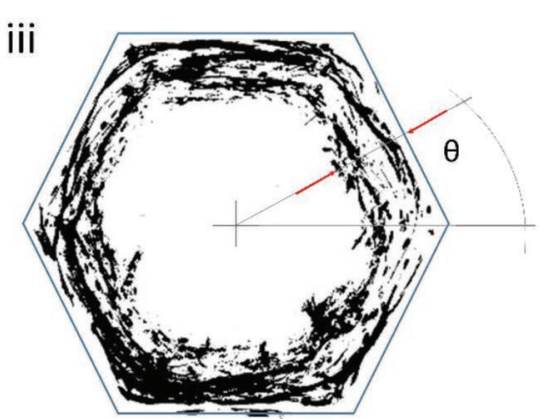

- Day 3 - Day 7

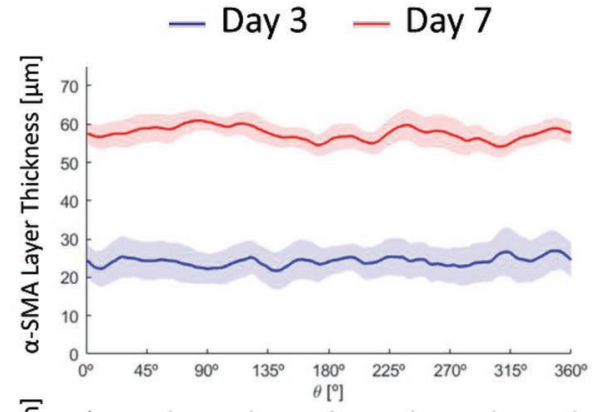

-SMA DAPI
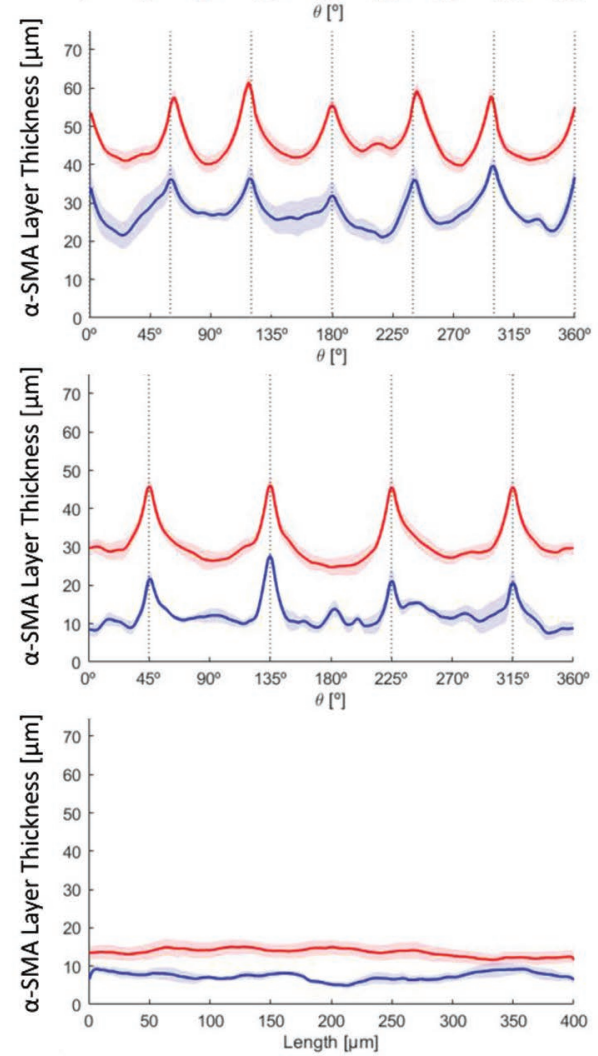

Figure 5. Distribution of $\alpha$-SMA-expressing SCs depends on compartment shape. A) Quantification process of $\alpha$-SMA distribution along the scaffold perimeter. (i) Images of vascular networks are obtained using confocal microscopy (scale bar: $100 \mu \mathrm{m})$. The red channel $(\alpha$-SMA) is separated for analysis. (ii) The red channel is binarized using a signal intensity threshold. (iii) Thickness of the $\alpha$-SMA signal is quantified in $\mu \mathrm{m}$ around the compartment walls. The angle $\theta\left[^{\circ}\right]$ is used to create a cartesian plot. B) Left: Representative confocal images of $\alpha$-SMA (red) distribution in the compartment geometries for day 3 and 7 (scale bar: $100 \mu \mathrm{m}$ ). The rightmost column shows representative images of the vascular networks inside the compartment for day 7 (green, HAMEC-ZsGreen; red, $\alpha$-SMA; blue, DAPI). Right: $\alpha$-SMA layer thickness on the compartment walls as a function of the angle $\theta$ for the circles, hexagon, and squares. For the rectangular compartments, the layer thickness is represented as a function of the length [ $\mu \mathrm{m}]$ along the wall. The blue and red plots represent the $\alpha$-SMA layer thickness at day 3 and 7, respectively. The soft shades of blue and red show the squared error of the mean $(n>5)$. 
polygons presented a particular pattern of peaks and valleys, associated with the corners and flat walls, respectively. Tessellated scaffolds with squared compartments had peaks located at $45^{\circ}, 135^{\circ}, 225^{\circ}$, and $315^{\circ}$, with a height of $21.44 \pm 2.85 \mu \mathrm{m}$ at day 3 , and nearly doubled by day 7 , with a mean height of $42.65 \pm 2.52 \mu \mathrm{m}$. The valleys, or flat sections, were located between the peaks. At day 3, scaffolds with squares presented a similar behavior to the scaffolds with rectangular compartments, with a mean thickness of $11.12 \pm 1.69 \mu \mathrm{m}$. However, by day 7 , the thinnest sections of the layer showed a mean thickness of $28.08 \pm 1.67 \mu \mathrm{m}$. Moreover, the valleys adopted a rounder curve, confirming our observations. The hexagon compartments displayed peaks around $0^{\circ}, 60^{\circ}, 120^{\circ}, 180^{\circ}, 240^{\circ}$, and $300^{\circ}$, coincident with the hexagon vertices. By day 3 , the valleys were more irregular than those of the square compartments, with a mean thickness of $24.81 \pm 3.51 \mu \mathrm{m}$; by day 7 , the valleys acquired a much rounder morphology, with a mean thickness of $45.15 \pm 3.35 \mu \mathrm{m}$. The width of the peaks increased from $33.26 \pm 2.84 \mu \mathrm{m}$ by day 3 to $55.62 \pm 2.71$ by day 7. As expected, the scaffolds with circular compartments displayed a uniformly distributed SMA layer around the wall, consistent with their radial symmetry. At day 3, the layer had a mean thickness of $24.17 \pm 6.24 \mu \mathrm{m}$, similar to the valleys of the hexagonal compartments at the same time point; by day 7, the mean thickness increased to $57.57 \pm 5.03 \mu \mathrm{m}$, resembling the mean value of the hexagon peaks at day 7 . The rectangular and circular shapes displayed a uniform thickness along the wall, suggesting an even SC-SMA+ distribution. On the other hand, both polygons exhibited highly uneven thickness values. The thinner layer sections were located around the center of the compartment walls, with a lower number of SCs-SMA+, while the thickest layers sections were situated at the corners, with a higher SC-SMA+ population.

Functional SCs modify their surroundings, especially when immersed in compliant substrates. ${ }^{[26]}$ We suspected that the uneven concentration of SCs-SMA+ in our system might generate distinct deformations of the hydrogel in accordance with their location. To monitor the displacements, fluorescent micrometric beads $(4 \mu \mathrm{m})$ were included in the fibrin gel. We imaged the hexagonal compartmented scaffolds during the first hours post SCs-seeding for $15 \mathrm{~h}$, with a frame interval of 30 min (Figure 6). We took confocal time-lapse images of the endothelialized scaffolds after adding SCs and microbeads (red) suspended in fibrin gel (Figure 6A). We binarized the fluorescent beads channel (Figure 6B) and compared the displacement of the beads from the first to last frame of the time lapse. The frames between the first and last one were used to obtain a smooth tracking of the micrometric displacements within the gel, allowing for continuous tracking and quantification. The displacements were represented as vector field, with the vector magnitude representing the displaced distance, and the vector direction showing the direction of the movement. Figure 6D displays the resulting vector field for the hexagonal compartments. Around the compartment wall, the vectors point inward, representing the gel constraining deformations exerted by the SCs. The maximum displacements are located on the compartment corners, while the vectors at the compartment center showed smaller displacements. This is consistent with the previous results, which showed the SCs predilection to concentrate on the compartment walls and, in particular, on the compartment corners. The more concentrated the SCs, the larger deformations they produce, as they proliferate, organize, and modify the ECM, assisting in the tubulogenesis phenomenon (Movie S3, Supporting Information).

\section{Discussion}

The physiological process of vessel network formation includes complex interactions between ECs and SCs. ${ }^{[27]}$ Engineering these networks in a controlled fashion requires a deep understanding of key factors governing the involved cells and formed vessels development. ${ }^{[1]}$ The environment in which the cells are immersed has an impact on the resulting network; previous works mostly focused on the geometry influence of free gels on vascular development, but with no consideration of the effect imparted by the pore geometries. ${ }^{[10-13,28]}$ Although hydrogels represent a key element in tissue engineering for cell growth, development, and reorganization, ${ }^{[29]}$ the addition of stiff and porous scaffolds is fundamental for mechanical support and reduces tissue shrinkage, especially when creating thick tissues. ${ }^{[30]}$ Thus, the scaffold effect cannot be ignored when studying vessel development. In this work, we intended to harness the scaffold effect on vessel networks to study sprouting decisions. To this purpose, we fabricated tessellated polymer scaffolds, consisting of identical repeating microenvironments, which enabled high-throughput analysis. Furthermore, the scaffolds can be designed in a wide range of shapes, according to the user's preference and the experiment purpose, providing an excellent environment for 3D imaging and real-time monitoring of cellular phenomena, and can be adapted to grow different cell types using various hydrogels. To produce engineered vessels, the traditional coculture method consists of simultaneously seeding ECs and SCs, but does not provide control over the sprouting locations. ${ }^{[31]}$ Alternatively, using a novel two-step cell seeding protocol, we achieved highly organized and homogeneously distributed vascular networks, with hollow vessels mimicking the compartment shape with high fidelity (Figure S3, Supporting Information). This is of particular relevance for creating microvascular networks that resemble intricate native structures, like those irrigating the nephron or hepatic units, which currently remain a great challenge. ${ }^{[32,33]}$ We suggest that by combining our two-step seeding protocol with precise scaffold fabrication techniques, such as 3D jet writing, stereolithography, or microextrusion-based $3 \mathrm{D}$ printing, ${ }^{[15,16,34]}$ it is possible to recreate the complex features of such intricate structures.

Compartment geometry had an impact on vessel thickness at early developmental stages (Figure 2B) and played a significant role in its organization; new sprouting vessels followed a specific orientation within polygonal compartments (hexagons and squares), connecting the center of the sides to opposing corners (Figure 3B). Real-time imaging showed that a higher percentage of ECs-formed tubular sprouts depart from the center of the polygon sides and evidenced a clear tendency to migrate toward the scaffold corners (Figure 4), with migration velocities depending on the compartment geometry (Figure S9, Supporting Information). We interpreted these phenomena as the vessel network behavior in relation to the compartment 

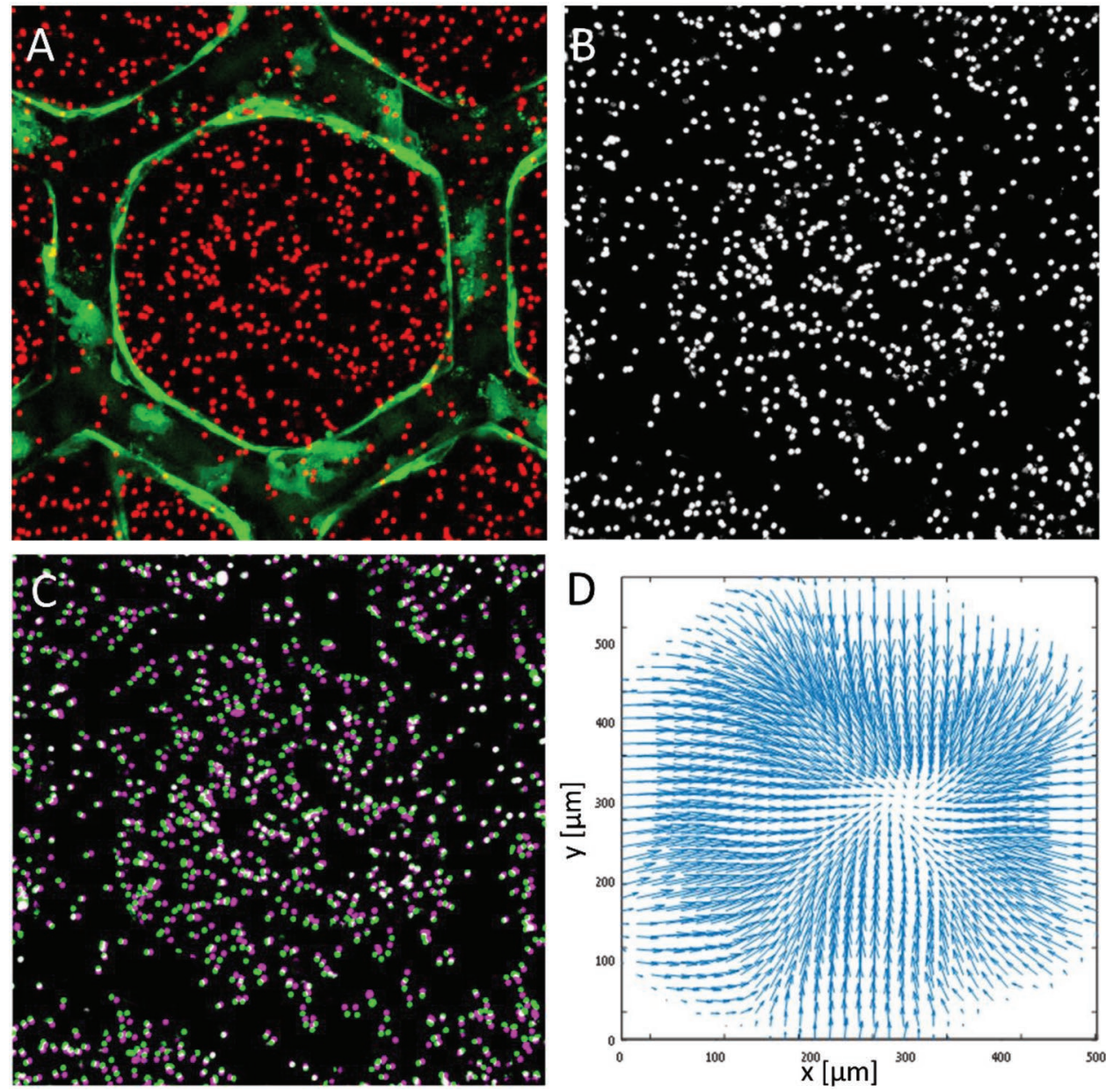

Figure 6. Displacement quantification in shaped compartments. A) Confocal image of ECs (green) commencing the tubulogenesis process within a hexagonal compartment. The fibrin used to seed the SCs (not shown) contained uniformly dispersed $4 \mu \mathrm{m}$ fluorescent beads (red). B) The binarized image of the beads is used to quantify displacements within the hydrogel. The images are obtained using real-time imaging, with frame intervals of $30 \mathrm{~min}$. C) An example of the bead movement between two frames. The green dots show the position of the beads in the first frame, the magenta dots represent the position of the beads in the last frame, and the white dot sections of the dots represent the bead overlaps between the first and last frames. D) Vector field of the gel displacements within a hexagonal compartment $(n=3)$. The vector length indicates the displacement magnitude, and the vector angle indicates the displacement direction of the beads within the gel.

topology, and thus hypothesized that the quantified origin and migration trends were related to SCs-SMA+ location and accumulation.

SCs affinity for stiffer surfaces caused an accumulation on the compartment walls, especially on the corners, where anchoring sites are more available (Figure 5B). We propose that this uneven SCs distribution may be the cause for the vessel orientations; the higher population of support cells located at the corners might create a chemotactic cytokines gradient, drawing the sprouting vessels. Previous works have shown that for different geometries, the highest cytokine expression by $\mathrm{SCs}$, such as vascular endothelial growth factor A (VEGF-A), is located at the corners, creating an inward gradient. ${ }^{[13]}$ VEGF-A is one of many angiogenic factors that cue new vessels to sprout and to migrate into a specific direction. ${ }^{[7]}$ We suggest that vessels sprouted more often from the center of the sides following the same principle. On the wall center, SCs-SMA+ numbers are at its lowest, decreasing their stabilizing effect on the vessels, allowing for increased sprouting and mobility. ${ }^{[7]}$
Therefore, the observed location of the SCs-SMA++ can explain the sprout orientation patterns within the compartments. Furthermore, this was observed in accordance with in vivo results that demonstrated the migration and concentration of blood vessels into concave surfaces. ${ }^{[35]}$ For the analyzed shapes, the mechanical micrometric displacements corresponded with the observed SCs-SMA+ distribution, as support cells are known to modify their surroundings and exert great strains on compliable matrices. ${ }^{[26,36]}$ Although both cell types were mostly localized on the walls during the hydrogel deformation measurements, we attributed the displacement effects to the support cells, since previous works have demonstrated that fibroblast-like cells have a higher impact in gel deformation than ECs. ${ }^{[37]}$ Moreover, the registered displacements are in accordance with the presence of chemotactic gradients, as higher gel strains were determined as a cause for higher SCs cytokine release. ${ }^{[13]}$ To generalize our findings, considering the presented work here and the cited bibliography, we hypothesize that the sprouting vessels and SC behavior shown in our 
research would also occur in more general setups consisting of stiff scaffolds embedded in softer hydrogels, regardless of the scaffold architecture.

We believe that understanding how to control the overall vasculature orientation and sprouting directions can lead to producing engineered constructs that better mimic the native tissue, especially when creating tissues with highly oriented networks, such as skeletal and cardiac muscle. ${ }^{[38-40]}$ Further, the system created in this study can provide crucial insight for improving scaffold design that might be extrapolated to other up-and-coming fabrication techniques, such as 3D bioprinting.

\section{Conclusion}

Here, we present a high-throughput platform that allows for controlling and organizing spontaneously formed microvascular networks. The system provides a highly suitable 3D environment for cell growth and tissue formation, and subsequent static or real-time imaging using a variety of tests. Our novel two-step seeding protocol allows for creating vascular networks that are capable of tracing compartment shapes with high fidelity. We showed that vascular networks respond to compartment geometry, evidencing differences in vessel morphology. Furthermore, compartment shape has a clear effect on the support cell behavior, encouraging higher concentration and proliferation in rounder shapes or corners. We propose that the SCs-SMA+ uneven distribution is a key factor behind sprouting decision; vessels emerged more frequently from areas with lower numbers of SCs-SMA+, and predominantly migrated into more populated areas in the compartment. Finally, we believe that our findings in sprouting decision making and scaffold compartment impact on vascular network morphology take us one step further into recreating native tissues.

\section{Experimental Section}

Cell Culture: HAMECs (ScienceCell) were cultured in endothelial cell medium bullet kit (ScienceCell, Cat. No. 1001). DPSCs (Lonza) were grown in low-glucose Dulbecco's modified Eagle medium (DMEM; Gibco), supplemented with $10 \%$ fetal bovine serum (FBS; Hyclone), $1 \%$ non-essential amino acids (NEAAs, Gibco, CATNUM), 1\% GlutaMAX (Gibco, catnumber), and 1\% penicillin-streptomycin-nystatin solution (Biological Industries). HAMECs passage 2 and DPSCs passage 2 were transduced to express ZsGreen and DtTomato fluorescent proteins, respectively, using lentiviral transfection. Cells were used until passage 9 and grown until $\approx 85 \%$ confluence before expansion or cell seeding.

Tessellated Scaffold Fabrication: Scaffolds were fabricated using photolithography technique. A 4 in. silicon wafer was spin-coated thrice with Omnicoat (Microchem Corp.), followed by a $1 \mathrm{~min}$ bake at $200{ }^{\circ} \mathrm{C}$ after each coat. The Omnicoat coatings acted as sacrificial layers, enabling lift-off of the scaffolds from the wafer. SU-8 2050 (Microchem Corp.) was spun onto the Omnicoat-coated silicon wafer until a thickness of $\approx 100 \mu \mathrm{m}$ was achieved. Then, the photoresist was exposed to UV light, as indicated by the manufacturer recommendation. The scaffolds were lifted off during the final development step and were subsequently washed with isopropyl alcohol for at least six times. Scaffolds were then dried for a minimum of one week before use.

Stepwise Cell Seeding: Tessellated scaffolds were dipped in ethanol for $15 \mathrm{~min}$ for sterilization, then washed with phosphate buffered saline (PBS). Then, scaffolds were covered with a fibronectin solution (50 $\mu \mathrm{g} \mathrm{mL} \mathrm{m}^{-1}$, Sigma) in PBS and incubated at $37{ }^{\circ} \mathrm{C}$ for $1 \mathrm{~h}$ and washed with PBS. HAMEC-ZsGreen were suspended in medium with a concentration of $4 \times 10^{6}$ cells $\mathrm{mL}^{-1}$, the fibronectin-coated scaffolds were placed in wells and covered with $25 \mu \mathrm{L}$ of the cell suspension and cultured in the incubator for $1 \mathrm{~h}$. Endothelial cell medium was added to the wells and cultured for $2 \mathrm{~d}$. Then, DPSCs were suspended in fibrin pre-gel solution $\left(1: 115 \mathrm{mg} \mathrm{mL}^{-1}\right.$ BAC2:5 $\mathrm{U} \mathrm{mL}^{-1}$ thrombin,

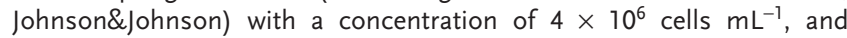
$25 \mu \mathrm{L}$ of fibrin were seeded to each pre-endothelialized scaffold. Fibrin was allowed to solidify for $30 \mathrm{~min}$ at $37^{\circ} \mathrm{C}$, and 1:1 ECs:SCs medium was added to the wells. The constructs were cultured for up to $7 \mathrm{~d}$, with medium changed every $2-3 \mathrm{~d}$.

Immunofluorescent Staining and Confocal Imaging: After 3 or $7 \mathrm{~d}$ of culturing, scaffolds were fixed using paraformaldehyde (4\%, PFA) for $20 \mathrm{~min}$, followed by $3 \times$ PBS washes, $5 \mathrm{~min}$ each. The cells were permeabilized using Tryton-X (0.3\%) for $15 \mathrm{~min}$ and washed three times with PBS. The constructs were blocked with bovine serum albumin (5\%, BSA, MilliPore) solution for $1 \mathrm{~h}$ at room temperature, and stained with von Willebran factor (1:150, VWF, AbCam) and smooth muscle actin (1:50, SMA, Dako) at $4{ }^{\circ} \mathrm{C}$ overnight. Constructs were then washed thrice with PBS, and the staining was completed using goat- $\alpha$ mouse Cy3 (1:150, Jackson Immunoresearch Laboratory) and goat- $\alpha$ rabbit Alexa-Fluor 488 (1:400, Thermo-Fisher Scientific). The live or fixed samples were imaged using a confocal microscope LSM 700 (Zeiss).

Scanning Electron Microscopy Imaging: Constructs were fixed using a silification protocol, as described by Kaehr et al. ${ }^{[41]}$ Briefly, the scaffolds or cultured constructs were fixated using PFA (4\%) for 20 min and washed with PBS. The samples were immersed in tetramethyl orthosilicate $\left(100 \times 10^{-3} \mathrm{M}\right.$, Sigma) and $\mathrm{HCl}$ solution $\left(1 \times 10^{-3} \mathrm{M}\right)$ for $4 \mathrm{~d}$ at $40{ }^{\circ} \mathrm{C}$. Then, the samples were dehydrated using sequential ethanol dilutions, $10 \mathrm{~min}$ each. The constructs were imaged using a Quanta 200 microscope (FEI).

Multicolor Endothelial Cell Seeding for Time-Lapse Tracking: To facilitate single sprout identification and tracking, the two-step cell seeding protocol was performed as usual. However, instead of seeding $4 \times 10^{6}$ cells $\mathrm{mL}^{-1}$ with HAMEC-ZsGreen, the seeding was performed using $1.33 \times 10^{6}$ cells $\mathrm{mL}^{-1}$ of HAMEC-ZsGreen, HAMEC-TdTomato, and DiD-stained HAMEC. After the DPSC seeding and fibrin gelation, the constructs were cultured in an enclosed chamber within the confocal microscope imaging path. The culture chamber was set with the same conditions as a regular incubator, that is, temperature of $37{ }^{\circ} \mathrm{C}$ and $5 \%$ $\mathrm{CO}_{2}$. Images were taken every $2 \mathrm{~h}$ for a period of $10 \mathrm{~d}$.

Fluorescent Beads Inclusion for Time-Lapse Strain Tracking: To measure inner gel displacements, $4 \mu \mathrm{m}$ red fluorescent beads (0.5\%, Invitrogen) were added to the fibrin gel during the support cell seeding. Time-lapse imaging was performed as explained above, although images were taken every $30 \mathrm{~min}$, for $3 \mathrm{~d}$.

Image Processing, Analysis, and Statistical Analysis: The obtained confocal images were processed using Image) to create maximum projection images, remove background noise, and modify gamma value (0.6). Vascular network development analysis was performed using Angiotool. ${ }^{[19]}$ For vessel orientation codification, total vessel length angle distribution, bead displacement measurement, and SMA quantification, ad hoc Matlab (MathWorks) scripts were written and implemented. Manual cell tracking was performed using the Manual Tracking plug-in in Image). Statistical analysis was performed using Prism (GraphPad Software). Significance differences among the groups were assessed using one-way analysis of variance (ANOVA), followed by Tukey's multiple comparison test. Differences were considered significant for $p<0.05$. All data are presented as mean \pm standard deviation.

\section{Supporting Information}

Supporting Information is available from the Wiley Online Library or from the author. 


\section{Acknowledgements}

This research was supported by funding from the University of Michigan - Israel Partnership for Research. The authors would like to thank Oryan Katovitz, Abigail Newman, Shahar Ben-Shaul, and Galia Ben-David for their great assistance and support.

\section{Conflict of Interest}

The authors declare no conflict of interest.

\section{Keywords}

compartment geometry, high throughput analysis, sprouting, tissue engineering, vascular development

Received: February 13, 2019

Revised: May 7, 2019

Published online: June 7, 2019

[1] E. C. Novosel, C. Kleinhans, P. J. Kluger, Adv. Drug Delivery Rev. 2011, 63, 300.

[2] S. Landau, A. A. Szklanny, G. C. Yeo, Y. Shandalov, E. Kosobrodova, A. S. Weiss, S. Levenberg, Biomaterials 2017, 122, 72.

[3] A. Lesman, J. Koffler, R. Atlas, Y. J. Blinder, Z. Kam, S. Levenberg, Biomaterials 2011, 32, 7856.

[4] S. Landau, S. Guo, S. Levenberg, Front. Bioeng. Biotechnol. 2018, 6, 2.

[5] Y. J. Blinder, D. J. Mooney, S. Levenberg, Curr. Opin. Chem. Eng. $2014,3,56$

[6] D. Richards, J. Jia, M. Yost, R. Markwald, Y. Mei, Ann. Biomed. Eng. 2017, 45, 132.

[7] M. Potente, H. Gerhardt, P. Carmeliet, Cell 2011, 146, 873.

[8] M. I. Gariboldi, R. Butler, S. M. Best, R. E. Cameron, PLoS One 2019, 14, e0210390.

[9] J. A. Henry, K. Burugapalli, P. Neuenschwander, A. Pandit, Acta Biomater. 2009, 5, 29.

[10] S. Raghavan, C. M. Nelson, J. D. Baranski, E. Lim, C. S. Chen, Tissue Eng., Part A 2010, 16, 2255.

[11] J. Sun, N. Jamilpour, F. Y. Wang, P. K. Wong, Biomaterials 2014, 35, 3273.

[12] P. Bose, J. Eyckmans, T. D. Nguyen, C. S. Chen, D. H. Reich, ACS Biomater. Sci. Eng. 2018, https://doi.org/10.1021/ acsbiomaterials.8b01183.

[13] N. C. Rivron, E. J. Vrij, J. Rouwkema, S. Le Gac, A. van den Berg, R. K. Truckenmuller, C. A. van Blitterswijk, Proc. Natl. Acad. Sci. USA 2012, 109, 6886 .

[14] R. Gauvin, Y.-C. Chen, J. W. Lee, P. Soman, P. Zorlutuna, J. W. Nichol, H. Bae, S. Chen, A. Khademhosseini, Biomaterials 2012, 33, 3824.

[15] S. Coscoy, S. Baiz, J. Octon, B. Rhoné, L. Perquis, Q. Tseng, F. Amblard, V. Semetey, Biointerphases 2018, 13, 041003.
[16] J. H. Jordahl, L. Solorio, H. Sun, S. Ramcharan, C. B. Teeple, H. R. Haley, K. J. Lee, T. W. Eyster, G. D. Luker, P. H. Krebsbach, J. Lahann, Adv. Mater. 2018, 30, 1707196.

[17] R. J. Klebe, K. L. Bentley, R. C. Schoen, J. Cell. Physiol. 1981, 109, 481.

[18] Y. J. Blinder, A. Freiman, N. Raindel, D. J. Mooney, S. Levenberg, Sci. Rep. 2016, 5, 17840.

[19] E. Zudaire, L. Gambardella, C. Kurcz, S. Vermeren, PLoS One 2011, 6, e27385.

[20] H. B. M. Uylings, Bull. Math. Biol. 1977, 39, 509.

[21] I. A. Lubashevsky, V. V. Gafiychuk, 1999, arXiv preprint adap-org/9909003.

[22] A. Chauviere, L. Preziosi, T. Hillen, Networks Heterog. Media 2007, 2, 333.

[23] H. El-Mohri, Y. Wu, S. Mohanty, G. Ghosh, Mater. Sci. Eng., C 2017, 74, 146.

[24] M. Guthold, W. Liu, E. A. Sparks, L. M. Jawerth, L. Peng, M. Falvo, R. Superfine, R. R. Hantgan, S. T. Lord, Cell Biochem. Biophys. 2007, 49, 165.

[25] K. Okeyo, H. Kotera, M. Washizu, $\mu$ TAS Proc. 2013, 17, 27.

[26] K. A. Jansen, R. G. Bacabac, I. K. Piechocka, G. H. Koenderink, Biophys. J. 2013, 105, 2240.

[27] J. Welti, S. Loges, S. Dimmeler, P. Carmeliet, J. Clin. Invest. 2013, 123,3190

[28] M. Nikkhah, N. Eshak, P. Zorlutuna, N. Annabi, M. Castello, K. Kim, A. Dolatshahi-Pirouz, F. Edalat, H. Bae, Y. Yang, A. Khademhosseini, Biomaterials 2012, 33, 9009.

[29] S. R. Caliari, J. A. Burdick, Nat. Methods 2016, 13, 405.

[30] S. J. Hollister, Nat. Mater. 2006, 5, 590.

[31] J. Rouwkema, A. Khademhosseini, Trends Biotechnol. 2016, 34, 733.

[32] M. J. Wilmer, C. P. Ng, H. L. Lanz, P. Vulto, L. Suter-Dick, R. Masereeuw, Trends Biotechnol. 2016, 34, 156.

[33] V. L. Tsang, A. A. Chen, L. M. Cho, K. D. Jadin, R. L. Sah, S. DeLong, J. L. West, S. N. Bhatia, FASEB J. 2007, 21, 790 .

[34] A. Panwar, L. P. Tan, Molecules 2016, 21, 685

[35] A. Scarano, V. Perrotti, L. Artese, M. Degidi, D. Degidi, A. Piattelli, G. lezzi, Odontology 2014, 102, 259.

[36] B. A. Juliar, M. T. Keating, Y. P. Kong, E. L. Botvinick, A. J. Putnam, Biomaterials 2018, 162, 99.

[37] T. Takebe, M. Enomura, E. Yoshizawa, M. Kimura, H. Koike, Y. Ueno, T. Matsuzaki, T. Yamazaki, T. Toyohara, K. Osafune, H. Nakauchi, H. Y. Yoshikawa, H. Taniguchi, Cell Stem Cell 2015, 16, 556.

[38] A. W. Feinberg, P. W. Alford, H. Jin, C. M. Ripplinger, A. A. Werdich, S. P. Sheehy, A. Grosberg, K. K. Parker, Biomaterials 2012, 33, 5732.

[39] S. Levenberg, J. Rouwkema, M. Macdonald, E. S. Garfein, D. S. Kohane, D. C. Darland, R. Marini, C. A. van Blitterswijk, R. C. Mulligan, P. A. D'Amore, R. Langer, Nat. Biotechnol. 2005, 23, 879.

[40] D. Rosenfeld, S. Landau, Y. Shandalov, N. Raindel, A. Freiman, E. Shor, Y. Blinder, H. H. Vandenburgh, D. J. Mooney, S. Levenberg, Proc. Natl. Acad. Sci. USA 2016, 113, 3215.

[41] B. Kaehr, J. L. Townson, R. M. Kalinich, Y. H. Awad, B. S. Swartzentruber, D. R. Dunphy, C. J. Brinker, Proc. Natl. Acad. Sci. USA 2012, 109, 17336 